\title{
3D Deformations by means of Monogenic Functions ${ }^{\dagger}$
}

\author{
J. Morais $\ddagger$ \\ Technical University of Mining, \\ Freiberg, Germany \\ Email: joao.pedro.morais@ua.pt
}

\author{
M. Ferreira \\ School of Technology and Management, \\ Polytechnic Institute of Leiria, Portugal \\ 2411-901 Leiria, Portugal. \\ Email: milton.ferreira@ipleiria.pt \\ and \\ CIDMA - Center for Research and Development \\ in Mathematics and Applications, \\ University of Aveiro, 3810-193 Aveiro, Portugal. \\ Email: mferreira@ua.pt
}

\begin{abstract}
In this paper the authors compute the coefficient of quasiconformality for monogenic functions in an arbitrary ball of the Euclidean space $\mathbb{R}^{3}$. This quantification may be needed in applications but also appear to be of intrinsic interest. The main tool used is a 3D Fourier series development of monogenic functions in terms of a special set of solid spherical monogenics. Ultimately, we present some examples showing the applicability of our approach.
\end{abstract}

MSC 2000: 30G35; 30C65

Keywords: Quaternion analysis; Riesz system; monogenic functions; quasiconformal mappings.

\section{Introduction}

By a classical theorem of Liouville [28], the usual definition of conformal mappings in the $n$-dimensional Euclidean space $\mathbb{R}^{n}$ when $n \geq 3$ applies only to the restricted set of Möbius transformations. For this reason, the theory of conformal mappings is essentially restricted to $2 \mathrm{D}$ settings. Actually, for quasiconformal mappings the situation is quite different; there exist several possible ways to give an infinitesimal notion of quasiconformality. In order to explain our standing position, let us consider a homeomorphism $f$ of a domain $\mathcal{D} \subset \mathbb{R}^{n}$ onto a domain $\mathcal{D}^{\prime} \subset \mathbb{R}^{n}$. For $x \in \mathcal{D}, r>0$ and a 3D closed ball $\bar{B}(x, r) \subset \mathcal{D}$ of center $x$ and radius $r$, we set

$$
L_{f}(x, r):=\max _{|x-y|=r}|f(y)-f(x)|, \quad l_{f}(x, r):=\min _{|x-y|=r}|f(y)-f(x)|, \quad k_{f}(x, r)=\frac{L_{f}(x, r)}{l_{f}(x, r)} .
$$

The coeffcient of quasiconformality (or linear dilatation) of $f$ at $x \in \mathcal{D}$ is defined as $k_{f}(x)=\lim _{\sup } \operatorname{su}_{r \rightarrow 0} k_{f}(x, r)$. For our purposes here, the orientation preserving homeomorphism $f: \mathcal{D} \rightarrow \mathcal{D}^{\prime}$ is called $k$-quasiconformal

\footnotetext{
${ }^{\dagger}$ Accepted author's manuscript (AAM) published in [Math. Meth. Appl. Sci., 36(7) (2013), 780-793] [DOI: 10.1002/mma.2625]. The final publication is available at link.springer.com via http://onlinelibrary.wiley.com/doi/10.1002/mma.2625/abstract

${ }^{\ddagger}$ Corresponding author
} 
in $\mathcal{D}^{\prime}$ if $k_{f}(x)$ is bounded in $\mathcal{D}$ and $k_{f}(x) \leq k$ for almost all points $x \in \mathcal{D}$. Then a mapping is called quasiconformal if it is $k$-quasiconformal for some $k(1 \leq k<\infty)$. The distortion theory of quasiconformal mappings is usually connected to problems of obtaining global distortion bounds under quasiconformal deformations from the local bounds of $k_{f}$. The major sources of the theory of quasiconformal mappings in the plane are found in the works of Grötzsch [14], Lavrent'ev [25], Ahlfors [1], and Teichmüller [41]. In the meantime, quasiconformal mappings became a classical object of analysis due to its rich behavior and wide range of applicability in various fields of mathematics such as discrete group theory, mathematical physics, complex differential geometry, medical image analysis, and probability theory. Higher dimensional quasiconformal mappings were first introduced by Lavrent'ev in 1938 [26], followed over a period of several years by a series of famous works: Ahlfors [2], Gehring [13], and Väisälä [42, 43]. The reader can consult the book of Rickman [37] for a more recent treatment of the subject.

In general, the study of quasiconformal mappings is important for the construction of analytic mappings with specified dynamics, which can be used as coordinate transformations in various problems, e.g. in the study of partial differential equations [7]. The dilatation of quasiconformal mappings controls the way conformal invariants, such as moduli of annuli, can be changed. Especially, in the theory of renormalizations, we can measure how distant a map is from another one in terms of dilatations of conjugacies. For that it is essential to have an estimate for the dilatation of the quasiconformal mapping. For such applications in higher dimensions the study of the quasiconformality factor is fundamental. Recent statistical studies have shown that quasi-conformal mappings can also be useful for approximate recovery of the boundary shape of domains in inverse problems, e.g. in scattering, diffraction problems and tomography [20, 21, 22, 23].

The discussion about the extension of theoretical and practical quasiconformal mapping techniques in the quaternion analysis setting, has originated many questions. Yet a large number of investigations $[12,15,24,29,40]$ were carried out in connection with the goal of studying monogenic functions by a corresponding differentiability concept or by the existence of a well-defined hypercomplex derivative. However, up to now there are feeble attempts to characterize monogenic functions via a generalized conformality concept. It was Malonek who first introduced the concept of monogenic conformal mappings [30]. These mappings are called in [30] $\mathcal{M}$-conformal mappings. The relation of this concept with the geometric interpretation of the hypercomplex derivative $\frac{1}{2} \bar{D}$ allowed it to complete the theory of monogenic functions by providing an accounting for the still missing geometric characterization of those functions. In [32] Malonek et al. studied the existence of local homeomorphims for quaternionic Beltrami-type equations, and determined a necessary and sufficient criterion that relates the hypercomplex derivative of a quaternion monogenic function and its corresponding Jacobian determinant. However, according to our current knowledge nothing has been done in the study of the quasiconformality factor.

Some of the recent interest in this subject was stimulated by the works of Gürlebeck et al. [16, 17, 18, 19] (cf. [35], Ch.4) in which it is proved that the class of monogenic functions with nonvanishing Jacobian determinant can be defined as a special subclass of quasi-conformal mappings. More precisely, it is shown that monogenic functions map locally balls onto explicitly characterized ellipsoids and vice versa. Besides this, methods used in [18] show that these considerations include the description of the interplay between the Jacobian determinant and the hypercomplex derivative of a monogenic function also. In continuation of these studies, our goal is to show how do the coefficient of quasiconformality looks like in the case of a monogenic function defined in a ball of $\mathbb{R}^{3}$ with values in the reduced quaternions (identified with $\mathbb{R}^{3}$ ). This is particularly rewarding since the computation of this coefficient will give us the information of the ratio of the major to minor axes of the aforementioned ellipsoids. In our approach, as we shall see, the key tool is the representation of a monogenic 3D Fourier series in 
terms of a special set of solid spherical monogenics. From our point of view these results can be seen as a step towards for a deeper understanding on the local behavior of monogenic mappings. Later, just as importantly, this can be used as a basis to study the global behavior of such mappings. In general, it is still open how to provide the description of monogenic functions via their global geometric mapping properties. The interest in questions of this type has increased in connection with constructing a theory of monogenic mappings. A first global result was considered recently by Almeida and Malonek in $[3,31]$ (cf. [11]). The authors have studied the global behavior of the higher dimensional analogue of the classical Joukowski transform in the context of Clifford analysis.

\section{Notation and definitions}

The present section collects some definitions and basic properties of quaternion analysis that will be needed throughout the text. Let $\mathbb{H}:=\left\{\mathbf{z}=z_{0}+z_{1} \mathbf{i}+z_{2} \mathbf{j}+z_{3} \mathbf{k}, z_{i} \in \mathbb{R}, i=0,1,2,3\right\}$ be the real quaternion algebra, where the imaginary units $\mathbf{i}, \mathbf{j}$, and $\mathbf{k}$ are subject to the multiplication rules:

$$
\mathbf{i}^{2}=\mathbf{j}^{2}=\mathbf{k}^{2}=-1 ; \quad \mathbf{i j}=\mathbf{k}=-\mathbf{j i}, \quad \mathbf{j k}=\mathbf{i}=-\mathbf{k j}, \quad \mathbf{k i}=\mathbf{j}=-\mathbf{i k} .
$$

As usual, the real vector space $\mathbb{R}^{4}$ may be embedded in $\mathbb{H}$ by identifying the element $z:=\left(z_{0}, z_{1}, z_{2}, z_{3}\right) \in$ $\mathbb{R}^{4}$ with $\mathbf{z}:=z_{0}+z_{1} \mathbf{i}+z_{2} \mathbf{j}+z_{3} \mathbf{k} \in \mathbb{H}$. Let us make a notation convention. Consider the subset $\mathcal{A}:=\operatorname{span}_{\mathbb{R}}\{1, \mathbf{i}, \mathbf{j}\}$ of $\mathbb{H}$, then the real vector space $\mathbb{R}^{3}$ may be embedded in $\mathcal{A}$ via the identification of $x:=\left(x_{0}, x_{1}, x_{2}\right) \in \mathbb{R}^{3}$ with the reduced quaternion $\mathbf{x}:=x_{0}+x_{1} \mathbf{i}+x_{2} \mathbf{j} \in \mathcal{A}$. To this end, throughout the text, we will often use the symbol $x$ to represent a point in $\mathbb{R}^{3}$ and $\mathbf{x}$ to represent the corresponding reduced quaternion. Also, it may be worthwhile to point out that $\mathcal{A}$ is a real vectorial subspace, but not a subalgebra of $\mathbb{H}$. Like in the complex case, $\mathbf{S c}(\mathbf{x})=x_{0}$ and $\mathbf{V e c}(\mathbf{x})=x_{1} \mathbf{i}+x_{2} \mathbf{j}$ define the scalar and vector parts of $\mathbf{x}$. The conjugate of $\mathbf{x}$ is the reduced quaternion $\overline{\mathbf{x}}=x_{0}-x_{1} \mathbf{i}-x_{2} \mathbf{j}$, and the norm $|\mathbf{x}|$ of $\mathbf{x}$ is defined by $|\mathbf{x}|^{2}=\mathbf{x} \overline{\mathbf{x}}=\overline{\mathbf{x}} \mathbf{x}=x_{0}^{2}+x_{1}^{2}+x_{2}^{2}$, and it coincides with its corresponding Euclidean norm as a vector in $\mathbb{R}^{3}$. Let $\Omega$ be an open subset of $\mathbb{R}^{3}$ with a piecewise smooth boundary. We say that $\mathbf{f}: \Omega \longrightarrow \mathcal{A}, \mathbf{f}(x)=[\mathbf{f}(x)]_{0}+[\mathbf{f}(x)]_{1} \mathbf{i}+[\mathbf{f}(x)]_{2} \mathbf{j}$ is a reduced quaternion-valued function or, in other words, an $\mathcal{A}$-valued function, where $[\mathbf{f}]_{i}(i=0,1,2)$ are real-valued functions defined in $\Omega$. Properties (like integrability, continuity or differentiability) that are ascribed to $\mathbf{f}$ have to be fulfilled by all components $[\mathbf{f}]_{i}$. We further introduce the real-linear Hilbert space of square integrable $\mathcal{A}$-valued functions defined in $\bar{B}(x, r)$, that we denote by $L_{2}(\bar{B}(x, r) ; \mathcal{A} ; \mathbb{R})$. The scalar inner product is defined by

$$
<\mathbf{f}, \mathbf{g}>_{L_{2}(\bar{B}(x, r) ; \mathcal{A} ; \mathbb{R})}=\int_{\bar{B}(x, r)} \mathbf{S c}(\overline{\mathbf{f}} \mathbf{g}) d V
$$

where $d V$ denotes the volume of $\bar{B}(x, r)$. For continuously real-differentiable $\mathcal{A}$-valued functions $\mathbf{f}$, the reader may be familiar with the (reduced) quaternionic operators $D=\partial_{x_{0}}+\mathbf{i} \partial_{x_{1}}+\mathbf{j} \partial_{x_{2}}$ and $\bar{D}=\partial_{x_{0}}-\mathbf{i} \partial_{x_{1}}-\mathbf{j} \partial_{x_{2}}$, which are called generalized Cauchy-Riemann (resp. conjugate generalized Cauchy-Riemann) operators on $\mathbb{R}^{3}$. Namely, a continuously real-differentiable $\mathcal{A}$-valued function $\mathbf{f}$ is said to be monogenic in $\bar{B}(x, r)$ if $D \mathbf{f}=0$ in $\bar{B}(x, r)$, which is equivalent to the system

$$
\left\{\begin{array}{l}
\frac{\partial[\mathbf{f}]_{0}}{\partial x_{0}}-\frac{\partial[\mathbf{f}]_{1}}{\partial x_{1}}-\frac{\partial[\mathbf{f}]_{2}}{\partial x_{2}}=0 \\
\frac{\partial[\mathbf{f}]_{0}}{\partial x_{1}}+\frac{\partial[\mathbf{f}]_{1}}{\partial x_{0}}=0, \quad \frac{\partial[\mathbf{f}]_{0}}{\partial x_{2}}+\frac{\partial[\mathbf{f}]_{2}}{\partial x_{0}}=0, \quad \frac{\partial[\mathbf{f}]_{1}}{\partial x_{2}}-\frac{\partial[\mathbf{f}]_{2}}{\partial x_{1}}=0
\end{array} .\right.
$$


We may point out that the previous system is called the Riesz system [38], and it generalizes the classical Cauchy-Riemann system in the plane. Following [27], the solutions of the system (R) are called (R)solutions. The subspace of polynomial (R)-solutions of degree $n$ will be denoted by $\mathcal{R}^{+}(\bar{B}(x, r) ; \mathcal{A} ; n)$. In [27], it is shown that the space $\mathcal{R}^{+}(\bar{B}(x, r) ; \mathcal{A} ; n)$ has dimension $2 n+3$. We also denote by $\mathcal{R}^{+}(\bar{B}(x, r) ; \mathcal{A}):=L_{2}(\bar{B}(x, r) ; \mathcal{A} ; \mathbb{R}) \cap \operatorname{ker} D$ the space of square integrable $\mathcal{A}$-valued monogenic functions defined in $\bar{B}(x, r)$.

For brevity, assume in the sequel that $\mathbf{f}$ is an $\mathcal{A}$-valued monogenic function. Furthermore, let $J_{\mathbf{f}}=\left(\partial_{x_{j}}[\mathbf{f}]_{i}\right)_{i, j=0}^{2}$ be the Jacobian of $\mathbf{f}$ and suppose that the mapping $\mathbf{f}$ preserves the orientation. There is a great difference in the properties of holomorphic conformal 2D-mappings and monogenic quasiconformal 3D-mappings. Methods used in [16, 17, 18, 19] and [35] Ch.4, provide us with an idea of the interaction between quasiconformality and monogenic functions. To be precise, every function f realizes locally in the neighborhood of a fixed point $x=x^{*}$ a $\mathcal{M}$-conformal mapping if and only if $\left.\operatorname{det} J_{\mathbf{f}}(x)\right|_{x=x^{*}} \neq 0$.

Theorem 1 (see [19]) Let $\mathbf{f}$ be an $\mathcal{A}$-valued real-analytic function defined in $\Omega$ with non-vanishing Jacobian determinant. Then, the function $\mathbf{f}$ is monogenic if and only if it maps locally a ball onto an ellipsoid with the property that the reciprocal of the length of one semi-axis is equal to the sum of the reciprocals of the lengths of the other two semi-axes.

Ultimately, we conclude this section by recalling a suitable set of special monogenic polynomials in the space $\mathcal{R}^{+}(\bar{B}(x, r) ; \mathcal{A} ; n)$, which was introduced in [8] (see also [9]) by applying the hypercomplex derivative $\frac{1}{2} \bar{D}$ (see $[15,34,40]$ ) to a standard system of spherical harmonics as considered e.g. in [39]. First, consider $\mathbf{x}$ with polar coordinates $\left(r, \theta_{1}, \theta_{2}\right): x_{0}=r \cos \theta_{1}, x_{1}=r \sin \theta_{1} \cos \theta_{2}$, and $x_{2}=$ $r \sin \theta_{1} \sin \theta_{2}$, where $0<r<\infty, 0<\theta_{1} \leq \pi$, and $0<\theta_{2} \leq 2 \pi$. To this end, we first select the set of homogeneous harmonic polynomials,

$$
\left\{U_{n+1}^{l, \dagger}, V_{n+1}^{m, \dagger}: l=0,1, \ldots, n+1, m=1, \ldots, n+1\right\}_{n \in \mathbb{N}_{0}}
$$

with the notations $U_{n+1}^{l, \dagger}:=r^{n+1} U_{n+1}^{l}$ and $V_{n+1}^{m, \dagger}:=r^{n+1} V_{n+1}^{m}$; it is formed by the extensions in the ball of the spherical harmonics $U_{n+1}^{l}\left(\theta_{1}, \theta_{2}\right)=P_{n+1}^{l}\left(\cos \theta_{1}\right) T_{l}\left(\cos \theta_{2}\right)(l=0, \ldots, n+1)$, and $V_{n+1}^{m}\left(\theta_{1}, \theta_{2}\right)=$ $P_{n+1}^{m}\left(\cos \theta_{1}\right) \sin \theta_{2} U_{m-1}\left(\cos \theta_{2}\right)(m=1, \ldots, n+1)$. Here, $P_{n+1}^{l}$ stands for the Ferrer's associated Legendre functions of degree $n+1$ and order $l$ of the first kind, $T_{l}$ and $U_{m-1}$ are the Chebyshev polynomials of the first and second kinds, respectively. We further assume the reader to be familiar with the fact that whenever $l=0$ the corresponding associated Legendre function $P_{n+1}^{0}$ coincides with the Legendre polynomial $P_{n+1}$, and $P_{n+1}^{l}$ are the zero functions for $l \geq n+2$. Based on the factorization of the 3D Laplace operator by $\Delta_{3}=D \bar{D}=\bar{D} D$, for each $n \in \mathbb{N}_{0}$ we do apply the operator $\frac{1}{2} \bar{D}$ to each basis element of (2). We obtain then the following set of $2 n+3$ homogeneous monogenic polynomials

$$
\left\{\mathbf{X}_{n}^{l, \dagger}, \mathbf{Y}_{n}^{m, \dagger}: l=0, \ldots, n+1, m=1, \ldots, n+1\right\}
$$

where $\mathbf{X}_{n}^{l, \dagger}:=r^{n} \mathbf{X}_{n}^{l}$ and $\mathbf{Y}_{n}^{m, \dagger}:=r^{n} \mathbf{Y}_{n}^{m}$. The fundamental references for these polynomials and their properties are [35] and [36]. The explicit expressions of the mentioned polynomials are given in the next proposition. 
Proposition 1 (see [36]) The homogenous monogenic polynomials (3) can be represented in the following way

$$
\begin{aligned}
& \mathbf{X}_{n}^{0, \dagger}:=r^{n}\left[\frac{(n+1)}{2} U_{n}^{0}+\frac{1}{2} U_{n}^{1} \mathbf{i}+\frac{1}{2} V_{n}^{1} \mathbf{j}\right] \\
& \mathbf{X}_{n}^{m, \dagger}:=r^{n}\left[\frac{(n+m+1)}{2} U_{n}^{m}+\frac{1}{4} R_{n}^{m,-} \mathbf{i}+\frac{1}{4} S_{n}^{m,+} \mathbf{j}\right] \\
& \mathbf{Y}_{n}^{m, \dagger}:=r^{n}\left[\frac{(n+m+1)}{2} V_{n}^{m}+\frac{1}{4} S_{n}^{m,-} \mathbf{i}-\frac{1}{4} R_{n}^{m,+} \mathbf{j}\right],
\end{aligned}
$$

with the notations $R_{n}^{m, \pm}\left(\theta_{1}, \theta_{2}\right):=U_{n}^{m+1} \pm(n+m+1)(n+m) U_{n}^{m-1}$, and $S_{n}^{m, \pm}\left(\theta_{1}, \theta_{2}\right):=V_{n}^{m+1} \pm(n+$ $m+1)(n+m) V_{n}^{m-1}$, where $m=1, \ldots, n+1$. For a more unified formulation we remind the reader that the spherical harmonics $U_{n}^{m}$ and $V_{n}^{m}$ are the zero function for $m \geq n+1$.

The next lemma shows that, for each $n \in \mathbb{N}_{0}$, the set (3) is composed by orthogonal polynomials with respect to the scalar inner product (1).

Lemma 1 (see $[8,9])$ For each $n=0,1, \ldots$, the polynomials $\mathbf{X}_{n}^{l, \dagger}(l=0, \ldots, n+1)$ and $\mathbf{Y}_{n}^{m, \dagger}(m=$ $1, \ldots, n+1)$ form a complete orthogonal system in $\mathcal{R}^{+}(\bar{B}(x, r) ; \mathcal{A})$, and their norms are explicitly given by

$$
\begin{aligned}
\left\|\mathbf{X}_{n}^{0, \dagger}\right\|_{L_{2}(\bar{B}(x, r) ; \mathcal{A} ; \mathbb{R})} & =\sqrt{\frac{r^{2 n+3}}{2 n+3} \pi(n+1)}, \\
\left\|\mathbf{X}_{n}^{m, \dagger}\right\|_{L_{2}(\bar{B}(x, r) ; \mathcal{A} ; \mathbb{R})} & =\left\|\mathbf{Y}_{n}^{m, \dagger}\right\|_{L_{2}(\bar{B}(x, r) ; \mathcal{A} ; \mathbb{R})}=\sqrt{\frac{r^{2 n+3}}{2 n+3} \frac{\pi}{2}(n+1) \frac{(n+1+m) !}{(n+1-m) !}} .
\end{aligned}
$$

Therefore, the Fourier series of $\mathbf{f}$ (centered at the origin) with respect to the referred orthogonal system in $\mathcal{R}^{+}(\bar{B}(x, r) ; \mathcal{A})$ is defined by

$$
\mathbf{f}=\sum_{n=0}^{\infty} \sqrt{\frac{2 n+3}{\pi(n+1) r^{2 n+3}}}\left[\mathbf{X}_{n}^{0, \dagger} a_{n}^{0}+\sum_{m=1}^{n+1} \sqrt{2 \frac{(n+1-m) !}{(n+1+m) !}}\left(\mathbf{X}_{n}^{m, \dagger} a_{n}^{m}+\mathbf{Y}_{n}^{m, \dagger} b_{n}^{m}\right)\right]
$$

where for each $n \in \mathbb{N}_{0}, a_{n}^{0}, a_{n}^{m}, b_{n}^{m}(m=1, \ldots, n+1)$ are the associated (real-valued) Fourier coefficients.

\section{The distance $|\mathbf{f}(y)-\mathbf{f}(x)|$ for $\mathcal{A}$-valued monogenic functions}

In this section we compute the coefficient of quasiconformality for any function defined in $\mathcal{R}^{+}(\bar{B}(x, r) ; \mathcal{A})$. For the reader's convenience and sake of easy reference, we will now briefly discuss this subject, following mainly the notations introduced in [33]. It should also be remarked that in some cases the idea is to work with the usual Taylor series expansion of a monogenic function in symmetric powers. We prefer here to consider a special monogenic Fourier series expansion in terms of homogenous monogenic polynomials.

To begin with, we shall find a general expression for the distance $|\mathbf{f}(y)-\mathbf{f}(x)|$. Whence, we consider $\mathbf{f}$ written as in (4). Since the basis polynomials $\mathbf{X}_{n}^{0, \dagger}, \mathbf{X}_{n}^{m, \dagger}$ and $\mathbf{Y}_{n}^{m, \dagger}$ are homogeneous we can write 
down an expansion of the form

$$
\begin{aligned}
\mathbf{f}(y)- & \mathbf{f}(x)=\sum_{n=0}^{\infty} \sqrt{\frac{2 n+3}{\pi(n+1) r^{2 n+3}}}\left\{\left(\mathbf{X}_{n}^{0, \dagger}(y)-\mathbf{X}_{n}^{0, \dagger}(x)\right) a_{n}^{0}\right. \\
& \left.+\sum_{m=1}^{n+1} \sqrt{2 \frac{(n+1-m) !}{(n+1+m) !}}\left[\left(\mathbf{X}_{n}^{m, \dagger}(y)-\mathbf{X}_{n}^{m, \dagger}(x)\right) a_{n}^{m}+\left(\mathbf{Y}_{n}^{m, \dagger}(y)-\mathbf{Y}_{n}^{m, \dagger}(x)\right) b_{n}^{m}\right]\right\} .
\end{aligned}
$$

A first straightforward computation shows that $|\mathbf{f}(y)-\mathbf{f}(x)|=\sqrt{\sum_{i=0}^{2}\left([\mathbf{f}(y)-\mathbf{f}(x)]_{i}\right)^{2}}=\sqrt{F_{0}^{2}+F_{1}^{2}+F_{2}^{2}}$, where

$$
\begin{gathered}
F_{0}:=[\mathbf{f}(y)-\mathbf{f}(x)]_{0}=\sum_{n=0}^{\infty} \sqrt{\frac{2 n+3}{\pi(n+1) r^{2 n+3}}}\left\{\frac{(n+1)}{2}\left(U_{n}^{0, \dagger}(y)-U_{n}^{0, \dagger}(x)\right) a_{n}^{0}\right. \\
\left.+\sum_{m=1}^{n} \sqrt{2 \frac{(n+1-m) !}{(n+1+m) !}} \frac{(n+1+m)}{2}\left[\left(U_{n}^{m, \dagger}(y)-U_{n}^{m, \dagger}(x)\right) a_{n}^{m}+\left(V_{n}^{m, \dagger}(y)-V_{n}^{m, \dagger}(x)\right) b_{n}^{m}\right]\right\} \\
F_{1}:=[\mathbf{f}(y)-\mathbf{f}(x)]_{1}=\sum_{n=0}^{\infty} \sqrt{\frac{2 n+3}{\pi(n+1) r^{2 n+3}}}\left\{\frac{1}{2}\left(U_{n}^{1, \dagger}(y)-U_{n}^{1, \dagger}(x)\right) a_{n}^{0}\right. \\
+\sum_{m=1}^{n+1} \sqrt{2 \frac{(n+1-m) !}{(n+1+m) !}} \frac{1}{4}\left[\left(U_{n}^{m+1, \dagger}(y)-U_{n}^{m+1, \dagger}(x)+(n+1+m)(n+m)\left(U_{n}^{m-1, \dagger}(x)-U_{n}^{m-1, \dagger}(y)\right)\right) a_{n}^{m}\right. \\
\left.\left.+\left(V_{n}^{m+1, \dagger}(y)-V_{n}^{m+1, \dagger}(x)+(n+1+m)(n+m)\left(V_{n}^{m-1, \dagger}(x)-V_{n}^{m-1, \dagger}(y)\right)\right) b_{n}^{m}\right]\right\}
\end{gathered}
$$

and

$$
\begin{aligned}
& F_{2}:=[\mathbf{f}(y)-\mathbf{f}(x)]_{2}=\sum_{n=0}^{\infty} \sqrt{\frac{2 n+3}{\pi(n+1) r^{2 n+3}}}\left\{\frac{1}{2}\left(V_{n}^{1, \dagger}(y)-V_{n}^{1, \dagger}(x)\right) a_{n}^{0}\right. \\
& +\sum_{m=1}^{n+1} \sqrt{2 \frac{(n+1-m) !}{(n+1+m) !}} \frac{1}{4}\left[\left(V_{n}^{m+1, \dagger}(y)-V_{n}^{m+1, \dagger}(x)+(n+1+m)(n+m)\left(V_{n}^{m-1, \dagger}(y)-V_{n}^{m-1, \dagger}(x)\right)\right) a_{n}^{m}\right. \\
& \left.\left.-\left(U_{n}^{m+1, \dagger}(y)-U_{n}^{m+1, \dagger}(x)+(n+1+m)(n+m)\left(U_{n}^{m-1, \dagger}(y)-U_{n}^{m-1, \dagger}(y)\right)\right) b_{n}^{m}\right]\right\} .
\end{aligned}
$$

The proof of the main result needs a series of technical preparations. Therefore, let us note the following lemma to our initial calculation:

Lemma 2 [35] The homogeneous harmonic polynomials $U_{n}^{l, \dagger}(l=0, \ldots, n)$ and $V_{n}^{m, \dagger}(m=1, \ldots, n)$ are given in Cartesian coordinates as:

$$
\begin{aligned}
U_{n}^{l, \dagger}(x) & =\sum_{k=0}^{\left[\frac{n-l}{2}\right]} \sum_{j=0}^{\left[\frac{l}{2}\right]} 2 \beta_{n, l, k} x_{0}^{n-2 k-l}|x|^{2 k}(-1)^{j}\left(\begin{array}{c}
l \\
2 j
\end{array}\right) x_{1}^{l-2 j} x_{2}^{2 j}, \\
V_{n}^{m, \dagger}(x) & =\sum_{k=0}^{\left[\frac{n-m}{2}\right]} \sum_{j=0}^{\left[\frac{m-1}{2}\right]} 2 \beta_{n, m, k} x_{0}^{n-2 k-m}|x|^{2 k}(-1)^{j}\left(\begin{array}{c}
m \\
2 j+1
\end{array}\right) x_{1}^{m-2 j} x_{2}^{2 j},
\end{aligned}
$$


where the upper bound $[s]$ denotes, as usual, the integer part of $s \in \mathbb{R}$ and the coefficients $\beta_{n, l, k}$ are defined in the following way

$$
\beta_{n, l, k}=(-1)^{k} \frac{1}{2^{n+1}}\left(\begin{array}{c}
2 n-2 k \\
n-k
\end{array}\right)\left(\begin{array}{c}
n-k \\
k
\end{array}\right)(n-2 k)_{l}
$$

Let us introduce a few abbreviations. In the sequel, we will make use of the following system of spherical coordinates:

$$
\begin{aligned}
& y_{0}=x_{0}+r \cos \theta_{1}=x_{0}+r c_{1} \\
& y_{1}=x_{1}+r \cos \theta_{2} \sin \theta_{1}=x_{1}+r c_{2} s_{1} \\
& y_{2}=x_{2}+r \sin \theta_{2} \sin \theta_{1}=x_{2}+r s_{2} s_{1}
\end{aligned}
$$

with $s_{i}=\sin \theta_{i}$ and $c_{i}=\cos \theta_{i}(i=1,2)$. This being so, we have

$$
\begin{aligned}
|y|^{2}=y_{0}^{2}+y_{1}^{2}+y_{2}^{2} & =\left(x_{0}+r c_{1}\right)^{2}+\left(x_{1}+r c_{2} s_{1}\right)^{2}+\left(x_{2}+r s_{2} s_{1}\right)^{2} \\
& =x_{0}^{2}+x_{1}^{2}+x_{2}^{2}+r^{2}+2 r\left(x_{0} c_{1}+x_{1} c_{2} s_{1}+x_{2} s_{1} s_{2}\right) \\
& =|x|^{2}+2 r \Lambda+r^{2},
\end{aligned}
$$

with $\Lambda=x_{0} c_{1}+x_{1} c_{2} s_{1}+x_{2} s_{1} s_{2}$. In cartesian coordinates it reads $\Lambda=1 / r\left(<x, y>-|x|^{2}\right)$. From this we have that

$$
\left(|y|^{2}\right)^{k}=\left(|x|^{2}+\left(2 r \Lambda+r^{2}\right)\right)^{k}=\sum_{h=0}^{k}\left(\begin{array}{c}
k \\
h
\end{array}\right)\left(|x|^{2}\right)^{k-h}\left(2 r \Lambda+r^{2}\right)^{h} .
$$

By defining the binomial function

$$
f_{h+q}:=\left(\begin{array}{c}
k \\
h
\end{array}\right)\left(\begin{array}{c}
h \\
q
\end{array}\right)\left(|x|^{2}\right)^{k-h}(2 \Lambda)^{h-q}
$$

satisfying $0 \leq h+q \leq 2 k, 0 \leq q \leq h \leq k$ and $f_{h+q}=0$ if $h>k$, we can rewrite the factor $\left(|y|^{2}\right)^{k}$ as

$$
\left(|y|^{2}\right)^{k}=\sum_{h+q=0}^{2 k} f_{h+q} r^{h+q} .
$$

Using the spherical coordinates (3) and the new relation (9) it is possible to write $U_{n}^{l, \dagger}$ and $V_{n}^{m, \dagger}$ as polynomial functions in $r$. Next we formulate the result.

Lemma 3 The polynomials $U_{n}^{l, \dagger}$ and $V_{n}^{m, \dagger}$ can be written in terms of the spherical coordinates (3) by

$$
U_{n}^{l, \dagger}(y)=\sum_{k=0}^{\left[\frac{n-l}{2}\right]} \sum_{j=0}^{\left[\frac{l}{2}\right]} 2 \beta_{n, l, k}(-1)^{j}\left(\begin{array}{c}
l \\
2 j
\end{array}\right) \sum_{t=0}^{n}\left(\sum_{p=0}^{t} g_{(k, p)}^{1} g_{(j, t-p)}^{2}\right) r^{t}
$$

with

$$
\begin{aligned}
& g_{\left(k, t_{1}\right)}^{1}:=\sum_{p=0}^{t_{1}}\left(\begin{array}{c}
n-2 k-l \\
p
\end{array}\right) x_{0}^{n-2 k-l-p} c_{1}^{p} f_{h+q=t_{1}-p}, \\
& g_{\left(j, t_{2}\right)}^{2}:=\sum_{p=0}^{t_{2}}\left(\begin{array}{c}
l-2 j \\
p
\end{array}\right)\left(\begin{array}{c}
2 j \\
t_{2}-p
\end{array}\right) c_{2}^{p} s_{1}^{t_{2}} s_{2}^{t_{2}-p} x_{1}^{l-2 j-p} x_{2}^{2 j-\left(t_{2}-p\right)}
\end{aligned}
$$


and

$$
V_{n}^{m, \dagger}(y)=\sum_{k=0}^{\left[\frac{n-m}{2}\right]} \sum_{j=0}^{\left[\frac{m-1}{2}\right]} 2 \beta_{n, m, k}(-1)^{j}\left(\begin{array}{c}
m \\
2 j+1
\end{array}\right) \sum_{t=0}^{n}\left(\sum_{p=0}^{t} h_{(k, p)}^{1} h_{(j, t-p)}^{2}\right) r^{t}
$$

where

$$
\begin{aligned}
& h_{\left(k, t_{1}\right)}^{1}=\sum_{p=0}^{t_{1}}\left(\begin{array}{c}
n-2 k-m \\
p
\end{array}\right) x_{0}^{n-2 k-m-p} c_{1}^{p} f_{h+q=t_{1}-p} \\
& h_{\left(j, t_{2}\right)}^{2}=\sum_{p=0}^{t_{2}}\left(\begin{array}{c}
m-2 j \\
p
\end{array}\right)\left(\begin{array}{c}
2 j \\
t_{2}-p
\end{array}\right) c_{2}^{p} s_{1}^{t_{2}} s_{2}^{t_{2}-p} x_{1}^{m-2 j-p} x_{2}^{2 j-\left(t_{2}-p\right)} .
\end{aligned}
$$

Proof: For sake of simplicity, we just present the proof of the expression (10). Using spherical coordinates (3) and (9), a first straightforward computation shows that

$$
\begin{aligned}
U_{n}^{l, \dagger}(y)= & \sum_{k=0}^{\left[\frac{n-l}{2}\right]} \sum_{j=0}^{\left[\frac{l}{2}\right]} 2 \beta_{n, l, k} y_{0}^{n-2 k-l}|y|^{2 k}(-1)^{j}\left(\begin{array}{c}
l \\
2 j
\end{array}\right) y_{1}^{l-2 j} y_{2}^{2 j} \\
= & \sum_{k=0}^{\left[\frac{n-l}{2}\right]} \sum_{j=0}^{\left[\frac{l}{2}\right]} 2 \beta_{n, l, k}\left(x_{0}+r c_{1}\right)^{n-2 k-l}\left(\sum_{h+q=0}^{2 k} f_{h+q} r^{h+q}\right) \\
& \times(-1)^{j}\left(\begin{array}{c}
l \\
2 j
\end{array}\right)\left(x_{1}+r c_{2} s_{1}\right)^{l-2 j}\left(x_{2}+r s_{1} s_{2}\right)^{2 j} .
\end{aligned}
$$

First we consider the expansions of the factors $\left(x_{0}+r c_{1}\right)^{n-2 k-l},\left(x_{1}+r c_{2} s_{1}\right)^{l-2 j}$ and $\left(x_{2}+r s_{1} s_{2}\right)^{2 j}$ :

$$
\begin{aligned}
\left(x_{0}+r c_{1}\right)^{n-2 k-l} & =\sum_{i_{1}=0}^{n-2 k-l}\left(\begin{array}{c}
n-2 k-l \\
i_{1}
\end{array}\right) x_{0}^{n-2 k-l-i_{1}}\left(r c_{1}\right)^{i_{1}}, \\
\left(x_{1}+r c_{2} s_{1}\right)^{l-2 j} & =\sum_{i_{2}=0}^{l-2 j}\left(\begin{array}{c}
l-2 j \\
i_{2}
\end{array}\right) x_{1}^{l-2 j-i_{2}}\left(r c_{2} s_{1}\right)^{i_{2}}, \\
\left(x_{2}+r s_{1} s_{2}\right)^{2 j} & =\sum_{i_{3}=0}^{2 j}\left(\begin{array}{c}
2 j \\
i_{3}
\end{array}\right) x_{2}^{2 j-i_{3}}\left(r s_{1} s_{2}\right)^{i_{3}} .
\end{aligned}
$$

Now, using the Cauchy product we obtain

$$
\left(x_{0}+r c_{1}\right)^{n-2 k-l}\left(\sum_{h+q=0}^{2 k} f_{h+q} r^{h+q}\right)=\sum_{t_{1}=0}^{n-l}\left(\sum_{p=0}^{t_{1}}\left(\begin{array}{c}
n-2 k-l \\
p
\end{array}\right) x_{0}^{n-2 k-l-p} c_{1}^{p} f_{h+q=t_{1}-p}\right) r^{t_{1}} .
$$

By defining

$$
g_{\left(k, t_{1}\right)}^{1}:=\sum_{p=0}^{t_{1}}\left(\begin{array}{c}
n-2 k-l \\
p
\end{array}\right) x_{0}^{n-2 k-l-p} c_{1}^{p} f_{h+q=t_{1}-p}
$$

we can write

$$
\left(x_{0}+r c_{1}\right)^{n-2 k-l}\left(\sum_{h+q=0}^{2 k} f_{h+q} r^{h+q}\right)=\sum_{t_{1}=0}^{n-l} g_{\left(k, t_{1}\right)}^{1} r^{t_{1}} .
$$


In (14) terms vanish whenever $p>n-2 k-l$ or $t_{1}-p>2 k$. The first restriction is satisfied by formula (14). For the second restriction we know that the binomial function $f_{h+q}$ is defined for $0 \leq h+q \leq 2 k$, being zero if $h+q<0$ or $h+q>2 k$. Therefore, the second restriction is also satisfied by formula (14). Similarly we obtain:

$$
\begin{aligned}
\left(x_{1}+r c_{2} s_{1}\right)^{l-2 j}\left(x_{2}+r s_{1} s_{2}\right)^{2 j} & =\sum_{i_{2}=0}^{l-2 j}\left(\begin{array}{c}
l-2 j \\
i_{2}
\end{array}\right) x_{1}^{l-2 j-i_{2}}\left(r c_{2} s_{1}\right)^{i_{2}} \sum_{i_{3}=0}^{2 j}\left(\begin{array}{c}
2 j \\
i_{3}
\end{array}\right) x_{2}^{2 j-i_{3}}\left(r s_{1} s_{2}\right)^{i_{3}} \\
& =\sum_{t_{2}=0}^{l}\left(\sum_{p=0}^{t_{2}}\left(\begin{array}{c}
l-2 j \\
p
\end{array}\right) x_{1}^{l-2 j-p}\left(c_{2} s_{1}\right)^{p}\left(\begin{array}{c}
2 j \\
t_{2}-p
\end{array}\right) x_{2}^{2 j-\left(t_{2}-p\right)}\left(s_{1} s_{2}\right)^{t_{2}-p}\right) r^{t_{2}} \\
& =\sum_{t_{2}=0}^{l}\left(\sum_{p=0}^{t_{2}}\left(\begin{array}{c}
l-2 j \\
p
\end{array}\right)\left(\begin{array}{c}
2 j \\
t_{2}-p
\end{array}\right) c_{2}^{p} s_{1}^{t_{2}} s_{2}^{t_{2}-p} x_{1}^{l-2 j-p} x_{2}^{2 j-\left(t_{2}-p\right)}\right) r^{t_{2}}
\end{aligned}
$$

By defining

$$
g_{\left(j, t_{2}\right)}^{2}:=\sum_{p=0}^{t_{2}}\left(\begin{array}{c}
l-2 j \\
p
\end{array}\right)\left(\begin{array}{c}
2 j \\
t_{2}-p
\end{array}\right) c_{2}^{p} s_{1}^{t_{2}} s_{2}^{t_{2}-p} x_{1}^{l-2 j-p} x_{2}^{2 j-\left(t_{2}-p\right)}
$$

we can write

$$
\left(x_{1}+r c_{2} s_{1}\right)^{l-2 j}\left(x_{2}+r s_{1} s_{2}\right)^{2 j}=\sum_{t_{2}=0}^{l} g_{\left(j, t_{2}\right)}^{2} r^{t_{2}} .
$$

In formula (15) terms vanish when $p>l-2 j$ or $t_{2}-p>2 j$. It is readily seen that these restrictions are encoded in formula (15). Finally, we compute the product between (14) and (15):

$$
\begin{aligned}
& \left(x_{0}+r c_{1}\right)^{n-2 k-l}\left(\sum_{h+q=0}^{2 k} f_{h+q} r^{h+q}\right)\left(x_{1}+r c_{2} s_{1}\right)^{l-2 j}\left(x_{2}+r s_{1} s_{2}\right)^{2 j} \\
& =\sum_{t_{1}=0}^{n-l} g_{\left(k, t_{1}\right)}^{1} r^{t_{1}} \sum_{t_{2}=0}^{l} g_{\left(j, t_{2}\right)}^{2} r^{t_{2}} \\
& =\sum_{t=0}^{n}\left(\sum_{p=0}^{t} g_{(k, p)}^{1} g_{(j, t-p)}^{2}\right) r^{t} .
\end{aligned}
$$

In formula (16) terms vanish when $p>n-l$ or $t-p>l$. Replacing (16) in (12) the polynomial $U_{n}^{l, \dagger}$ is finally given by

$$
U_{n}^{l, \dagger}(y)=\sum_{k=0}^{\left[\frac{n-l}{2}\right]} \sum_{j=0}^{\left[\frac{l}{2}\right]} 2 \beta_{n, l, k}(-1)^{j}\left(\begin{array}{c}
l \\
2 j
\end{array}\right) \sum_{t=0}^{n}\left(\sum_{p=0}^{t} g_{(k, p)}^{1} g_{(j, t-p)}^{2}\right) r^{t}
$$

In the same way, it can be proved the expression (11) for the polynomials $V_{n}^{m, \dagger}$.

We are now ready to proceed to the aim of the present paper, which consists of obtaining expressions for $F_{0}, F_{1}$, and $F_{2}$ in series of $r$. By Lemma 3 and straightforward computations we can compute the following differences:

$$
\begin{aligned}
U_{n}^{0, \dagger}(y)-U_{n}^{0, \dagger}(x) & =\sum_{k=0}^{\left[\frac{n}{2}\right]} 2 \beta_{n, 0, k} \sum_{t=1}^{n}\left(\sum_{p=0}^{t} \sum_{p_{1}=0}^{p}\left(\begin{array}{c}
n-2 k \\
p_{1}
\end{array}\right) x_{0}^{n-2 k-p_{1}} c_{1}^{p_{1}} f_{h+q=p-p_{1}}\right) r^{t} \\
& =\sum_{t=1}^{n} \sum_{k=0}^{\left[\frac{n}{2}\right]} 2 \beta_{n, 0, k}\left(\sum_{p=0}^{t} \sum_{p_{1}=0}^{p}\left(\begin{array}{c}
n-2 k \\
p_{1}
\end{array}\right) x_{0}^{n-2 k-p_{1}} c_{1}^{p_{1}} f_{h+q=p-p_{1}}\right) r^{t},
\end{aligned}
$$




$$
\begin{aligned}
& U_{n}^{m, \dagger}(y)-U_{n}^{m, \dagger}(x)= \\
& =\sum_{t=1}^{n} \sum_{k=0}^{\left[\frac{n-m}{2}\right]\left[\frac{m}{2}\right]} \sum_{j=0}^{t-p} 2 \beta_{n, m, k}(-1)^{j}\left(\begin{array}{c}
m \\
2 j
\end{array}\right) \sum_{p=0}^{t}\left(\sum_{p_{2}=0}^{p}\left(\begin{array}{c}
n-2 k-m \\
p_{2}
\end{array}\right) x_{0}^{n-2 k-m-p_{2}} c_{1}^{p_{2}} f_{h+q=p-p_{2}}\right) \\
& \left(\sum_{p_{3}=0}^{t-p}\left(\begin{array}{c}
m-2 j \\
p_{3}
\end{array}\right)\left(\begin{array}{c}
2 j \\
t-p-p_{3}
\end{array}\right) c_{2}^{p_{3}} s_{1}^{t-p} s_{2}^{t-p-p_{3}} x_{1}^{m-2 j-p_{3}} x_{2}^{2 j-\left(t-p-p_{3}\right)}\right) r^{t}
\end{aligned}
$$

and, moreover

$$
\begin{aligned}
& V_{n}^{m, \dagger}(y)-V_{n}^{m, \dagger}(x)= \\
& =\sum_{t=1}^{n} \sum_{k=0}^{\left[\frac{n-m}{2}\right]\left[\frac{m-1}{2}\right]} \sum_{j=0}^{2} 2 \beta_{n, m, k}(-1)^{j}\left(\begin{array}{c}
m \\
2 j+1
\end{array}\right) \sum_{p=0}^{t}\left(\sum_{p_{4}=0}^{p}\left(\begin{array}{c}
n-2 k-m \\
p_{4}
\end{array}\right) x_{0}^{n-2 k-m-p_{4}} c_{1}^{p_{4}} f_{h+q=p-p_{4}}\right) \\
& \left(\begin{array}{c}
t-p \\
\sum_{5}=0
\end{array}\left(\begin{array}{c}
m-2 j \\
p_{5}
\end{array}\right)\left(\begin{array}{c}
2 j \\
t-p-p_{5}
\end{array}\right) c_{2}^{p_{5}} s_{1}^{t-p} s_{2}^{t-p-p_{5}} x_{1}^{m-2 j-p_{5}} x_{2}^{2 j-\left(t-p-p_{5}\right)}\right) r^{t} .
\end{aligned}
$$

Replacing (17), (18), and (19) in (5), (6), and (7) we obtain

$$
F_{0}=\sum_{n=1}^{\infty} \sum_{t=1}^{n} f_{0,(k, j, t)}^{(n)} r^{t}, \quad F_{1}=\sum_{n=1}^{\infty} \sum_{t=1}^{n} f_{1,(k, j, t)}^{(n)} r^{t}, \quad F_{2}=\sum_{n=1}^{\infty} \sum_{t=1}^{n} f_{2,(k, j, t)}^{(n)} r^{t},
$$

with

$$
\begin{aligned}
& f_{0,(k, j, t)}^{(n)}=\sqrt{\frac{2 n+3}{\pi(n+1)}}\left\{\frac{(n+1)}{2}\left(\widetilde{\mathbf{U}}_{n}^{0, \dagger}(y)-\widetilde{\mathbf{U}}_{n}^{0, \dagger}(x)\right) a_{n}^{0}\right. \\
& \left.\quad+\sum_{m=1}^{n} \sqrt{2 \frac{(n+1-m) !}{(n+1+m) !}} \frac{(n+1+m)}{2}\left[\left(\widetilde{\mathbf{U}}_{n}^{m, \dagger}(y)-\widetilde{\mathbf{U}}_{n}^{m, \dagger}(x)\right) a_{n}^{m}+\left(\widetilde{\mathbf{V}}_{n}^{m, \dagger}(y)-\widetilde{\mathbf{V}}_{n}^{m, \dagger}(x)\right) b_{n}^{m}\right]\right\} .
\end{aligned}
$$

For the remaining factors, we have

$$
\begin{aligned}
& f_{1,(k, j, t)}^{(n)}=\sqrt{\frac{2 n+3}{\pi(n+1)}}\left\{\frac{1}{2}\left(\widetilde{\mathbf{U}}_{n}^{1, \dagger}(y)-\widetilde{\mathbf{U}}_{n}^{1, \dagger}(x)\right) a_{n}^{0}\right. \\
& +\sum_{m=1}^{n+1} \sqrt{2 \frac{(n+1-m) !}{(n+1+m) !}} \frac{1}{4}\left[\left(\widetilde{\mathbf{U}}_{n}^{m+1, \dagger}(y)-\widetilde{\mathbf{U}}_{n}^{m+1, \dagger}(x)+(n+1+m)(n+m)\left(\widetilde{\mathbf{U}}_{n}^{m-1, \dagger}(x)-\widetilde{\mathbf{U}}_{n}^{m-1, \dagger}(y)\right)\right) a_{n}^{m}\right. \\
& \left.\left.+\left(\widetilde{\mathbf{V}}_{n}^{m+1, \dagger}(y)-\widetilde{\mathbf{V}}_{n}^{m+1, \dagger}(x)+(n+1+m)(n+m)\left(\widetilde{\mathbf{V}}_{n}^{m-1, \dagger}(x)-\widetilde{\mathbf{V}}_{n}^{m-1, \dagger}(y)\right)\right) b_{n}^{m}\right]\right\}
\end{aligned}
$$

and,

$$
\begin{aligned}
& f_{2,(k, j, t)}^{(n)}=\sqrt{\frac{2 n+3}{\pi(n+1)}}\left\{\frac{1}{2}\left(\widetilde{\mathbf{V}}_{n}^{1, \dagger}(y)-\widetilde{\mathbf{V}}_{n}^{1, \dagger}(x)\right) a_{n}^{0}\right. \\
& +\sum_{m=1}^{n+1} \sqrt{2 \frac{(n+1-m) !}{(n+1+m) !}} \frac{1}{4}\left[\left(\widetilde{\mathbf{V}}_{n}^{m+1, \dagger}(y)-\widetilde{\mathbf{V}}_{n}^{m+1, \dagger}(x)+(n+1+m)(n+m)\left(\widetilde{\mathbf{V}}_{n}^{m-1, \dagger}(x)-\widetilde{\mathbf{V}}_{n}^{m-1, \dagger}(y)\right)\right) a_{n}^{m}\right. \\
& \left.\left.-\left(\widetilde{\mathbf{U}}_{n}^{m+1, \dagger}(y)-\widetilde{\mathbf{U}}_{n}^{m+1, \dagger}(x)+(n+1+m)(n+m)\left(\widetilde{\mathbf{U}}_{n}^{m-1, \dagger}(x)-\widetilde{\mathbf{U}}_{n}^{m-1, \dagger}(y)\right)\right) b_{n}^{m}\right]\right\}
\end{aligned}
$$


where for any $m=1, \ldots, n+1$

$$
\begin{aligned}
& \widetilde{\mathbf{U}}_{n}^{0, \dagger}(y)-\widetilde{\mathbf{U}}_{n}^{0, \dagger}(x)=\sum_{k=0}^{\left[\frac{n}{2}\right]} 2 \beta_{n, 0, k}\left(\sum_{p=0}^{t} \sum_{p_{1}=0}^{p}\left(\begin{array}{c}
n-2 k \\
p_{1}
\end{array}\right) x_{0}^{n-2 k-p_{1}} c_{1}^{p_{1}} f_{h+q=p-p_{1}}\right), \\
& \widetilde{\mathbf{U}}_{n}^{m, \dagger}(y)-\widetilde{\mathbf{U}}_{n}^{m, \dagger}(x)= \\
& =\sum_{k=0}^{\left[\frac{n-m}{2}\right]\left[\frac{m}{2}\right]} \sum_{j=0}^{t-p} 2 \beta_{n, m, k}(-1)^{j}\left(\begin{array}{c}
m \\
2 j
\end{array}\right) \sum_{p=0}^{t}\left(\sum_{p_{2}=0}^{p}\left(\begin{array}{c}
n-2 k-m \\
p_{2}
\end{array}\right) x_{0}^{n-2 k-m-p_{2}} c_{1}^{p_{2}} f_{h+q=p-p_{2}}\right) \\
& \left(\sum_{p_{3}=0}^{t-p}\left(\begin{array}{c}
m-2 j \\
p_{3}
\end{array}\right)\left(\begin{array}{c}
2 j \\
t-p-p_{3}
\end{array}\right) c_{2}^{p_{3}} s_{1}^{t-p} s_{2}^{t-p-p_{3}} x_{1}^{m-2 j-p_{3}} x_{2}^{2 j-\left(t-p-p_{3}\right)}\right)
\end{aligned}
$$

and

$$
\begin{aligned}
& \widetilde{\mathbf{V}}_{n}^{m, \dagger}(y)-\widetilde{\mathbf{V}}_{n}^{m, \dagger}(x)=
\end{aligned}
$$

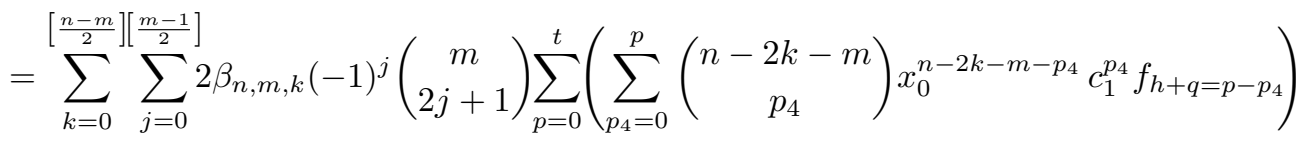

$$
\begin{aligned}
& \left(\sum_{p_{5}=0}^{t-p}\left(\begin{array}{c}
m-2 j \\
p_{5}
\end{array}\right)\left(\begin{array}{c}
2 j \\
t-p-p_{5}
\end{array}\right) c_{2}^{p_{5}} s_{1}^{t-p} s_{2}^{t-p-p_{5}} x_{1}^{m-2 j-p_{5}} x_{2}^{2 j-\left(t-p-p_{5}\right)}\right) \text {. }
\end{aligned}
$$

Since the series (20) are absolutely convergent we can write the functions $F_{0}, F_{1}$, and $F_{2}$ as polynomials functions in $r$, namely

$$
F_{0}=\sum_{t=1}^{\infty} \sum_{n=t}^{\infty} f_{0,(k, j, t)}^{(n)} r^{t}, \quad F_{1}=\sum_{t=1}^{\infty} \sum_{n=t}^{\infty} f_{1,(k, j, t)}^{(n)} r^{t}, \quad F_{2}=\sum_{t=1}^{\infty} \sum_{n=t}^{\infty} f_{2,(k, j, t)}^{(n)} r^{t},
$$

Now, considering the square of the functions $F_{0}, F_{1}$ and $F_{2}$ we obtain

$$
F_{0}^{2}=\sum_{p=1}^{\infty} a_{0, p} r^{p+1}, \quad F_{1}^{2}=\sum_{p=1}^{\infty} a_{1, p} r^{p+1}, \quad F_{2}^{2}=\sum_{p=1}^{\infty} a_{2, p} r^{p+1},
$$

where

$$
a_{i, p}:=\sum_{u=1}^{p} \sum_{n=u}^{\infty} f_{i,(k, j, u)}^{(n)} \sum_{v=p-u+1}^{\infty} f_{i,(k, j, p-u+1)}^{(v)}, \quad i=0,1,2 .
$$

Thus, it follows

$$
F_{0}^{2}+F_{1}^{2}+F_{2}^{2}=\sum_{p=1}^{\infty} A_{p} r^{p+1}
$$

where $A_{p}:=a_{0, p}+a_{1, p}+a_{2, p}$. In a compact form we finally obtain

$$
|f(y)-f(x)|=\sqrt{F_{0}^{2}+F_{1}^{2}+F_{2}^{2}}=\sqrt{\sum_{p=1}^{\infty} A_{p} r^{p+1}} .
$$




\section{Expansion factor and estimation of the quasiconformality factor}

In this section we give explicitly the coefficient of quasiconformality of any function defined in $\mathcal{R}^{+}(\bar{B}(x, r) ; \mathcal{A})$ depending on its Fourier coefficients. Next we formulate the result.

Theorem 2 (On the coefficient of quasiconformality) Let $\mathbf{f}$ be an $\mathcal{A}$-valued function defined in $\mathcal{R}^{+}(\bar{B}(x, r) ; \mathcal{A})$. The quasiconformality factor $k_{f}(x)$ is given by

$$
k_{f}(x):=\sqrt{\frac{\max A_{1}}{\min A_{1}}},
$$

where

$$
A_{1}=a_{0,1}+a_{1,1}+a_{2,1}, \quad a_{i, 1}=\sum_{n=1}^{\infty} f_{i,(k, j, 1)}^{(n)} \sum_{v=1}^{\infty} f_{i,(k, j, 1)}^{(v)} \quad(i=0,1,2) .
$$

Proof: For a given $\mathcal{A}$-valued monogenic function, we have proved in the previous section that

$$
|\mathbf{f}(y)-\mathbf{f}(x)|=\sqrt{\sum_{p=1}^{\infty} A_{p} r^{p+1}}=r \sqrt{A_{1}+A_{2} r+\ldots+A_{n} r^{n-1}+\ldots} .
$$

Taking the limit $r \rightarrow 0$ we obtain

$$
\lim _{r \rightarrow 0}\left[A_{1}+A_{2} r+\ldots+A_{n} r^{n-1}+\ldots\right]=A_{1} .
$$

By using the definition of quasiconformality

$$
k_{f}(x)=\limsup _{r \rightarrow 0} k_{f}(x, r)=\limsup _{r \rightarrow 0} \frac{L_{f}(x, r)}{l_{f}(x, r)},
$$

we can conclude that

$$
k_{f}(x)=\limsup _{r \rightarrow 0} \frac{\max \sqrt{A_{1}+A_{2} r+\ldots A_{n} r^{n-1}+\ldots}}{\min \sqrt{A_{1}+A_{2} r+\ldots A_{n} r^{n-1}+\ldots}}
$$

that gives an estimation for the factor $k$ of the quasiconformality of $f$, namely

$$
k_{f}(x):=\sqrt{\frac{\max A_{1}}{\min A_{1}}}
$$

where

$$
A_{1}=a_{0,1}+a_{1,1}+a_{2,1}, \quad a_{i, 1}=\sum_{n=1}^{\infty} f_{i,(k, j, 1)}^{(n)} \sum_{v=1}^{\infty} f_{i,(k, j, 1)}^{(v)} \quad(i=0,1,2) .
$$

For the expressions $f_{i,(k, j, 1)}^{(n)}, i=0,1,2$, considering $t=1$ in (21), (22), and (23) we obtain:

$$
\begin{aligned}
& \tilde{U}_{n}^{0, \dagger}(y)-\tilde{U}_{n}^{0, \dagger}(x)=\sum_{k=0}^{\left[\frac{n}{2}\right]} 2 \beta_{n, 0, k}\left(x_{0}^{n-2 k}|x|^{2 k}+2 k x_{0}^{n-2 k}\left(|x|^{2}\right)^{k-1} \Lambda+(n-2 k) x_{0}^{n-2 k-1} c_{1}|x|^{2 k}\right) \\
& \tilde{U}_{n}^{m, \dagger}(y)-\tilde{U}_{n}^{m, \dagger}(x)= \\
& =\sum_{k=0}^{\left[\frac{n-m}{2}\right]} \sum_{j=0}^{\left[\frac{m}{2}\right]} 2 \beta_{n, m, k}(-1)^{j}\left(\begin{array}{l}
m \\
2 j
\end{array}\right)\left[\left(x_{0}^{n-2 k-m}|x|^{2 k}\right)\left(2 j s_{1} s_{2} x_{1}^{m-2 j} x_{2}^{2 j-1}+(m-2 j) c_{2} s_{1} x_{1}^{m-2 j-1} x_{2}^{2 j}\right)\right. \\
& \left.+\left(2 k x_{0}^{n-2 k-m}\left(|x|^{2}\right)^{k-1} \Lambda+(n-2 k-m) x_{0}^{n-2 k-m-1} c_{1}|x|^{2 k}\right)\left(2 j x_{1}^{m-2 j} x_{2}^{2 j}\right)\right]
\end{aligned}
$$


and, moreover

$$
\begin{aligned}
& \tilde{V}_{n}^{m, \dagger}(y)-\tilde{V}_{n}^{m, \dagger}(x)= \\
& =\sum_{k=0}^{\left[\frac{n-m}{2}\right]} \sum_{j=0}^{\left.\frac{m-1}{2}\right]} 2 \beta_{n, m, k}(-1)^{j}\left(\begin{array}{c}
m \\
2 j+1
\end{array}\right)\left[\left(x_{0}^{n-2 k-m}|x|^{2 k}\right)\left(2 j s_{1} s_{2} x_{1}^{m-2 j} x_{2}^{2 j-1}+(m-2 j) c_{2} s_{1} x_{1}^{m-2 j-1} x_{2}^{2 j}\right)+\right. \\
& \left.+\left(2 k x_{0}^{n-2 k-m}\left(|x|^{2}\right)^{k-1} \Lambda+(n-2 k-m) x_{0}^{n-2 k-m-1} c_{1}|x|^{2 k}\right)\left(2 j x_{1}^{m-2 j} x_{2}^{2 j}\right)\right],
\end{aligned}
$$

where $\Lambda=x_{0} c_{1}+x_{1} c_{2} s_{1}+x_{2} s_{1} s_{2}$.

\section{Examples}

In this section we will compute the quasiconformality constant for some basis polynomials given in (3). For degree 1 , there is only one quasiconformal monogenic polynomial, which is $\mathbf{X}_{1}^{0, \dagger}=x_{0}+\frac{x_{1}}{2} \mathbf{i}+\frac{x_{2}}{2} \mathbf{j}$. For this polynomial we obtain $A_{1}=\frac{1}{2} \sqrt{4 \cos ^{2}\left(\theta_{1}\right)+\sin ^{2}\left(\theta_{1}\right)}$, and, therefore, $k_{\mathbf{X}_{1}^{0, \dagger}}(x)=\sqrt{2}$, independent of the point $x \in \mathbb{R}^{3}$ chosen. The other monogenic polynomials of degree 1 are not quasiconformal since $k_{f}(x)=0$. For example, for $\mathbf{X}_{1}^{1, \dagger}=\frac{3 x_{1}}{2}-\frac{3 x_{0}}{2} \mathbf{i}$ we obtain $A_{1}=\frac{3}{2} \sqrt{\cos ^{2}\left(\theta_{2}\right) \sin ^{2}\left(\theta_{1}\right)+\cos ^{2}\left(\theta_{1}\right)}$. Since in this case the minimum is zero, we have $k_{\mathbf{X}_{1}^{1, \dagger}}(x)=\infty$. For degree 2 there are some monogenic polynomials that are quasiconformal in some region of $\mathbb{R}^{3}$. For example, for the polynomial

$$
\mathbf{X}_{2}^{0, \dagger}=\frac{6 x_{0}^{2}-3 x_{1}^{2}-3 x_{2}^{2}}{4}+\frac{3 x_{0} x_{1}}{2} \mathbf{i}+\frac{3 x_{0} x_{2}}{2} \mathbf{j}
$$

we obtain

$$
\begin{aligned}
A_{1}= & \frac{3}{4}\left(4 x_{2}^{2}-4 x_{2}^{2} \cos ^{2}\left(\theta_{2}\right) \sin ^{2}\left(\theta_{1}\right)-8 x_{0} x_{2} \cos \left(\theta_{1}\right) \sin \left(\theta_{2}\right) \sin \left(\theta_{1}\right)+4 x_{0}^{2} \sin ^{2}\left(\theta_{1}\right)\right. \\
& -8 x_{0} x_{1} \cos \left(\theta_{1}\right) \cos \left(\theta_{2}\right) \sin \left(\theta_{1}\right)+4 x_{1}^{2}-4 x_{1}^{2} \sin ^{2}\left(\theta_{2}\right) \sin ^{2}\left(\theta_{1}\right)+16 x_{0}^{2} \cos ^{2}\left(\theta_{1}\right) \\
& \left.+8 x_{1} x_{2} \cos \left(\theta_{2}\right) \sin ^{2}\left(\theta_{1}\right) \sin \left(\theta_{2}\right)\right)^{\frac{1}{2}} .
\end{aligned}
$$

Using the computer algebra system Maple, we find that the maximum and the mininum of (32), for each point $x$ are given by

$$
\begin{aligned}
\max A_{1} & =\frac{3}{4}\left[\left(216\left|x_{0}\right|\left(x_{0}^{4}+\left(x_{1}^{2}+x_{2}^{2}\right) x_{0}^{2}+\frac{7}{27}\left(x_{1}^{2}+x_{2}^{2}\right)^{2}\right) \sqrt{9 x_{0}^{2}+4 x_{1}^{2}+4 x_{2}^{2}}\right.\right. \\
& \left.+648\left(x_{0}^{4}+\frac{7}{9} x_{0}^{2}\left(x_{1}^{2}+x_{2}^{2}\right)+\frac{1}{9}\left(x_{1}^{2}+x_{2}^{2}\right)^{2}\right)\left(x_{0}^{2}+\frac{4}{9}\left(x_{1}^{2}+x_{2}^{2}\right)\right)\right) / \\
& \left.\left(\left(4 x_{2}^{2}+4 x_{1}^{2}+9 x_{0}^{2}-3\left|x_{0}\right| \sqrt{9 x_{0}^{2}+4 x_{1}^{2}+4 x_{2}^{2}}\right)\left(9 x_{0}^{2}-3\left|x_{0}\right| \sqrt{9 x_{0}^{2}+4 x_{1}^{2}+4 x_{2}^{2}}+2 x_{1}^{2}+2 x_{2}^{2}\right)\right)\right]^{\frac{1}{2}}
\end{aligned}
$$

and

$$
\min A_{1}=\frac{3}{2}\left|x_{0}\right|
$$


Therefore, for $x_{0} \neq 0$ the quasiconformality factor $k_{\mathbf{X}_{2}^{0, \dagger}}(x)$ can be computed by (30). From (34) we can see that the quasiconformality constant $k_{\mathbf{X}_{2}^{0, \dagger}}(x)$ behaves as $\mathrm{O}\left(\frac{1}{\left|x_{0}\right|}\right)$. For $x_{0}=0$ the polynomial (31) is no longer quasiconformal.

In the next table we compute the quasiconformality factor $k_{\mathbf{X}_{2}^{0, \dagger}}(x)$ in different points:

\begin{tabular}{|c|c|}
\hline$x=\left(x_{0}, x_{1}, x_{2}\right)$ & $k_{\mathbf{X}_{2}^{0, \dagger}}(x)$ \\
\hline$\left(\frac{1}{2}, \frac{1}{2}, \frac{1}{2}\right)$ & 1.600485190 \\
\hline$\left(-1, \frac{1}{5}, 2\right)$ & 1.734354994 \\
\hline$(2,-3,5)$ & 1.943892809 \\
\hline$\left(\frac{1}{10}, \frac{1}{5}, 3\right)$ & 5.532086985 \\
\hline$\left(\frac{1}{100}, 1,0\right)$ & 10.02552988 \\
\hline
\end{tabular}

For degree 3 or higher there are also monogenic polynomials that are quasiconformal in some region of $\mathbb{R}^{3}$. We proceed with the monogenic polynomial of degree 3

$$
\mathbf{X}_{3}^{0, \dagger}=-3 x_{0} x_{2}^{2}-3 x_{0} x_{1}^{2}+2 x_{0}^{3}+\left(3 x_{0}^{2} x_{1}-\frac{3}{4} x_{1}^{3}-\frac{3}{4} x_{1} x_{2}^{2}\right) \mathbf{i}+\left(-\frac{3}{4} x_{1}^{2} x_{2}-\frac{3}{4} x_{2}^{3}+3 x_{0}^{2} x_{2}\right) \mathbf{j} .
$$

For this polynomial we obtain

$$
\begin{aligned}
A_{1}= & {\left[\left(81 x_{2}^{4}+90\left(x_{1}^{2}+4 x_{0}^{2}\right) x_{2}^{2}+9\left(x_{1}-2 x_{0}\right)^{2}\left(x_{1}+2 x_{0}\right)^{2}\right) \sin ^{2}\left(\theta_{2}\right)\right.} \\
& +144 \cos \left(\theta_{2}\right) x_{2} x_{1}\left(x_{1}^{2}+x_{2}^{2}+6 x_{0}^{2}\right) \sin \left(\theta_{2}\right)+9 \cos ^{2}\left(\theta_{2}\right)\left(x_{2}^{4}+\left(10 x_{1}^{2}-8 x_{0}^{2}\right) x_{2}^{2}+40 x_{0}^{2} x_{1}^{2}\right. \\
& \left.\left.+9 x_{1}^{4}+16 x_{0}^{4}\right)\right] \sin \left(\theta_{1}\right)^{2}+144\left(x_{2}^{2}+x_{1}^{2}-4 x_{0}^{2}\right) \cos \left(\theta_{1}\right) x_{0}\left(\sin \left(\theta_{2}\right) x_{2}+x_{1} \cos \left(\theta_{2}\right)\right) \sin \left(\theta_{1}\right) \\
& +144 \cos ^{2}\left(\theta_{1}\right)\left(4 x_{0}^{4}+x_{2}^{4}+2 x_{2}^{2} x_{1}^{2}+x_{1}^{4}\right)
\end{aligned}
$$

Using the computer algebra system Maple, we find that the maximum and the mininum of (36), for each point $x$, are given by

$$
\begin{aligned}
& \max A_{1}=9\left(\left(x_{1}^{2}+x_{2}^{2}+\frac{12}{7} x_{0}^{2}\right)\left(\left(x_{1}^{2}+x_{2}^{2}\right)^{2}-\frac{16}{9} x_{0}^{4}\right) \sqrt{49 x_{1}^{4}+88 x_{0}^{2} x_{1}^{2}+98 x_{1}^{2} x_{2}^{2}+144 x_{0}^{4}+88 x_{0}^{2} x_{2}^{2}+49 x_{2}^{4}}\right. \\
& +7 x_{2}^{8}+\left(\frac{128}{7} x_{0}^{2}+28 x_{1}^{2}\right) x_{2}^{6}+\left(42 x_{1}^{4}+\frac{384}{7} x_{0}^{2} x_{1}^{2}+\frac{3040}{63} x_{0}^{4}\right) x_{2}^{4} \\
& \left.+\left(\frac{2048}{63} x_{0}^{6}+\frac{384}{7} x_{0}^{2} x_{1}^{4}+28 x_{1}^{6}+\frac{6080}{63} x_{0}^{4} x_{1}^{2}\right) x_{2}^{2}+\frac{256}{7} x_{0}^{8}+\frac{3040}{63} x_{0}^{4} x_{1}^{4}+\frac{128}{7} x_{0}^{2} x_{1}^{6}+\frac{2048}{63} x_{0}^{6} x_{1}^{2}+7 x_{1}^{8}\right)^{\frac{1}{2}} / \\
& \quad\left[4 \left(\left(x_{1}^{2}+x_{2}^{2}-\frac{12}{7} x_{0}^{2}\right) \sqrt{49 x_{1}^{4}+88 x_{0}^{2} x_{1}^{2}+98 x_{1}^{2} x_{2}^{2}+144 x_{0}^{4}+88 x_{0}^{2} x_{2}^{2}+49 x_{2}^{4}}+7 x_{2}^{4}+\left(-\frac{40}{7} x_{0}^{2}+14 x_{1}^{2}\right) x_{2}^{2}\right.\right. \\
& \left.\left.\quad+7 x_{1}^{4}-\frac{40}{7} x_{0}^{2} x_{1}^{2}+\frac{144}{7} x_{0}^{4}\right)\right]
\end{aligned}
$$

and

$$
\min A_{1}=\frac{3}{4}\left|4 x_{0}^{2}-x_{1}^{2}-x_{2}^{2}\right|
$$

From (38) we can see that the minimum of $A_{1}$ is zero along the cone $x_{0}^{2}=\frac{x_{1}^{2}+x_{2}^{2}}{4}$. Since in this cone the maximum of $A_{1}$ is non-zero we conclude that the quasiconformality factor $k_{\mathbf{X}_{3}^{0, \dagger}}(x)$ in this case behaves as $O\left(\frac{1}{\left|4 x_{0}^{2}-x_{1}^{2}-x_{2}^{2}\right|}\right)$. For $x_{0}^{2}=\frac{x_{1}^{2}+x_{2}^{2}}{4}$ the polynomial (35) is no longer quasiconformal. In the next table we compute the quasiconformality factor $k_{\mathbf{X}_{3}^{0, \dagger}}(x)$ in different points: 


\begin{tabular}{|c|c|}
\hline$x=\left(x_{0}, x_{1}, x_{2}\right)$ & $k_{\mathbf{X}_{3}^{0, \dagger}}(x)$ \\
\hline$\left(\frac{1}{2}, \frac{1}{2}, \frac{1}{2}\right)$ & 2.485741007 \\
\hline$\left(-1, \frac{1}{5}, 2\right)$ & 21.21482516 \\
\hline$(2,-3,5)$ & 2.626259389 \\
\hline$\left(\frac{1}{10}, \frac{1}{5}, 3\right)$ & 1.737541999 \\
\hline$\left(\frac{1}{100}, 1,0\right)$ & 1.732545819 \\
\hline
\end{tabular}

Next figures show the deformation of the ball $\bar{B}(x, r)$, centered at $x$ with radius $r$, by the polynomials (31) and (35). Figures 1 to 3 correspond to the deformation by the polynomial (31) and Figures 4 to 6 correspond to the deformation by the polynomial (35). In Figures 1, 2, 4, and 5 we can observe that the ball is mapped onto an ellipsoid, but in figures 3 and 6 the ball is not mapped onto an ellipsoid since in the considered ball the mappings are not longer quasiconformal.
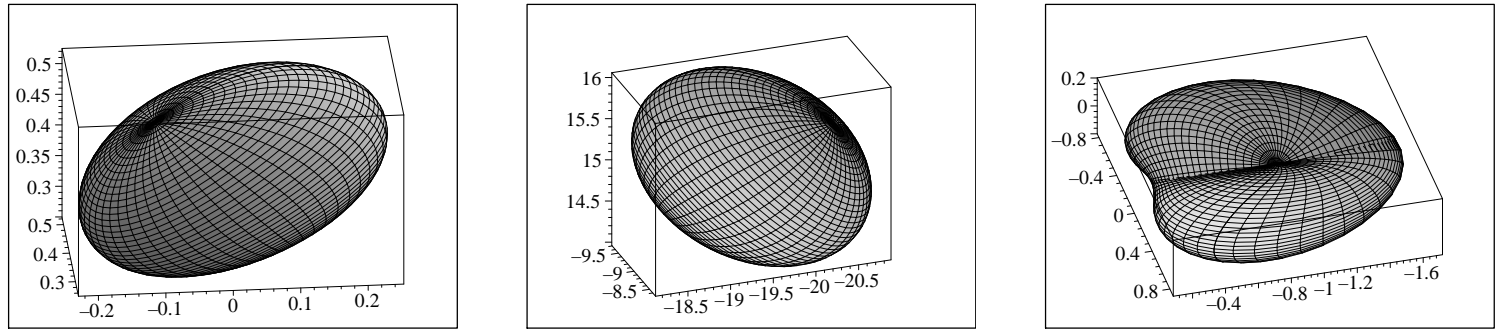

Fig. 1: $x=\left(\frac{1}{2}, \frac{1}{2}, \frac{1}{2}\right), r=\frac{1}{8}$

Fig. 2: $x=(2,-3,5), r=\frac{1}{8}$

Fig. 3: $x=\left(\frac{1}{100}, 1,0\right), r=\frac{1}{2}$
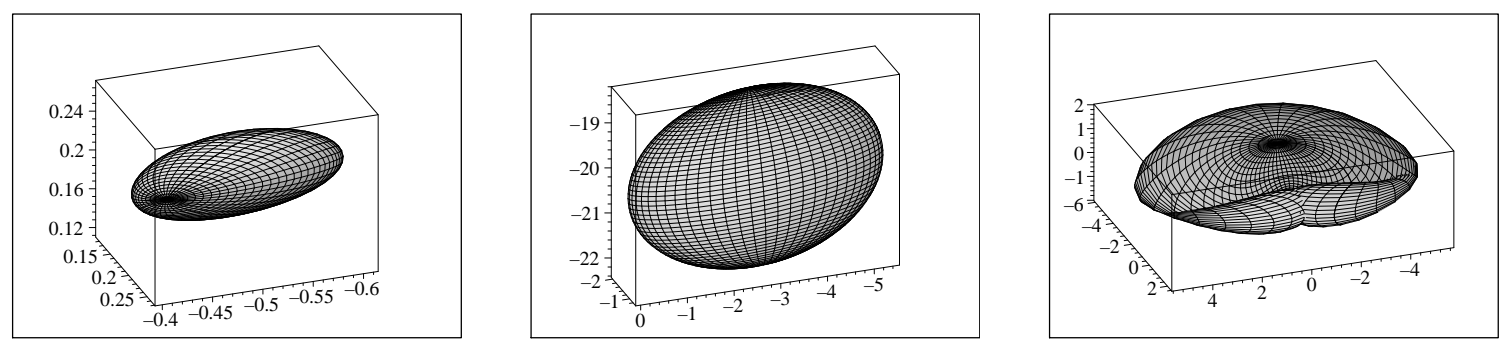

Fig. 4: $x=\left(\frac{1}{2}, \frac{1}{2}, \frac{1}{2}\right), r=\frac{1}{20}$

Fig. 5: $x=\left(\frac{1}{10}, \frac{1}{5}, 3\right), r=\frac{1}{10}$

Fig. 6: $x=\left(\frac{1}{100}, 1,0\right), r=1$

\section{Acknowledgements}

The first author acknowledges financial support from the Foundation for Science and Technology (FCT) via the post-doctoral grant SFRH/BPD/66342/2009. The second author's research is supported by FEDER funds through COMPETE-Operational Programme Factors of Competitiveness ("Programa Operacional Factores de Competitividade"i £ji £i) and by Portuguese founds through the Center for Research and Development in Mathematics and Applications (University of Aveiro) and the Portuguese Foundation for Science and Technology ("FCT-Fundação para a Ciência e a Tecnologia"i£¡ï£i), within project PEst-C/MAT/UI4106/2011 with COMPETE number FCOMP-01-0124-FEDER-022690.

\section{References}

[1] L. V. Ahlfors. Zur Theorie der Überlagerungsflächen, Acta Math. 65 (1935), 157-194.

[2] L. V. Ahlfors. Lectures on quasiconformal mappings, Van Nostrand, 1966. 
[3] R. Almeida and H. Malonek. On a Higher Dimensional Analogue of the Joukowski Transformation. AIP Conf. Proc. 1048, 630-633 (2008).

[4] G. D. Anderson, M. K. Varnanamurthy and M. Vuorinen. Dimension-free quasiconformal distortion in n-space, Trans. Amer. Math. Soc. 297 (1986), 687-706.

[5] G. D. Anderson, M. K. Varnanamurthy and M. Vuorinen. Sharp distortion theorems for quasiconformal mappings, Trans. Amer. Math. Soc. 305 (1988), 95-111.

[6] G. D. Anderson, M. K. Varnanamurthy and M. Vuorinen. Inequalities for quasiconformal mappings in the plane and space, Manuscript (1991), 1-43.

[7] K. Astala, T. Iwaniec and G. Martin. Elliptic partial differential equations and quasiconformal mappings in the plane, Vol. 48 of Princeton Mathematical Series, Princeton University Press, Princeton, NJ, 2009.

[8] I. Cação. Constructive approximation by monogenic polynomials. Ph.D. thesis, Universidade de Aveiro, Departamento de Matemática, 2004.

[9] I. Cação I, K. Gürlebeck and S. Bock. Complete Orthonormal Systems of Spherical Monogenics - A Constructive Approach. L. H. Son et al. (ed.), Methods of Complex and Clifford Analysis, SAS International Publications, Delhi (2005), 241-260.

[10] P. Caraman, n-dimensional quasiconformal mappings, Editura Academiei Romhne; Bucharest, Abacus Press;Tunebridge Wells Haessner Publishing, Inc.; Newfound- land, New Jersey, 1974.

[11] C. Cruz, M. I. Falcão, H. Malonek. 3D Mappings by Generalized Joukowski Transformations. Computational Science and Its Applications - ICCSA 2011, Lecture Notes in Computer Science, Volume 6784/2011, 358-373, Santander (2011).

[12] R. Delanghe, R. Kraußhar and H. Malonek. Differentiability of functions with values in some real associative algebras: approaches to an old problem. Bulletin de la Société Royale des Sciences de Liège, Vol. 70, No. 4-6, 231-249 (2001).

[13] F. W. Gehring. Quasiconformal mappings in space, Bull. Amer. Math. Soc. Volume 69, Number 2 (1963), 146-164.

[14] H. Grötzsch. Über möglichst konforme Abbildungen yon schlichten Bereichen, Ber. Verh. Sächs. Akad. Wiss. Leipzig 84 (1932), 114-120.

[15] K. Gürlebeck and H. Malonek. A hypercomplex derivative of monogenic functions in $\mathbb{R}^{n+1}$ and its applications. Complex Variables 39, 199-228 (1999).

[16] K. Gürlebeck and J. Morais. On mapping properties of monogenic functions, CUBO A Mathematical Journal, Vol. 11, No. 1, pp. 73-100 (2009).

[17] K. Gürlebeck and J. Morais. On Local Mapping Properties of Monogenic Functions, K. Gürlebeck and C. Könke (Editors): Proceedings 18th International Conference on the Applications of Computer Science and Mathematics in Architecture and Civil Engineering, Weimar (2009).

[18] K. Gürlebeck and J. Morais. Local properties of monogenic mappings, AIP Conference Proceedings, "Numerical analysis and applied mathematics", pp. 797-800 (2009).

[19] K. Gürlebeck and J. Morais. Geometric characterization of M-conformal mappings, Geometric Algebra Computing: in Engineering and Computer Science, Bayro-Corrochano, Eduardo; Scheuermann, Gerik (Eds.), Springer, 1st Edition, pp. 327-342, 2010.

[20] V. Kolehmainen, M. Lassas and P. Ola. The inverse conductivity problem with an imperfectly known boundary, SIAM Journal on Applied Mathematics, 66 (2005), pp. 365-383 (electronic).

[21] V. Kolehmainen, M. Lassas and P. Ola. Electrical impedance tomography problem with inaccurately known boundary and contact impedances, Medical Imaging, IEEE Transactions on, 27 (2008), pp. 1404-1414. 
[22] V. Kolehmainen, M. Lassas and P. Ola. Calderón's inverse problem with an imperfectly known boundary and reconstruction up to a conformal deformation, SIAM J. Math. Anal., 42 (2010), pp. 1371-1381.

[23] V. Kolehmainen, M. Lassas, P. Ola and S. Siltanen. Recovering boundary shape and conductivity in electrical impedance tomography, Inverse Problems and Imaging, (2012).

[24] R. Kraußhar and H. Malonek. A characterization of conformal mappings in $\mathbb{R}^{4}$ by a formal differentiability condition. Bulletin de la Société Royale des Sciences de Liège, Vol. 70, No. 1, 35-49 (2001).

[25] M. A. Lavrent'ev. Sur une cIasse de representatations continues, Mat. Sb. 42 (1935), 407-427.

[26] M. A. Lavrent'ev. Sur un critère differentiel des transformations homéomorphes des domains à trois dimensions, Dokl. Acad. Nank SSSR 20 (1938), 241-242.

[27] H. Leutwiler, Quaternionic analysis in $\mathbb{R}^{3}$ versus its hyperbolic modification, Brackx, F., Chisholm, J.S.R. and Soucek, V. (ed.). NATO Science Series II. Mathematics, Physics and Chemistry, vol. 25, Kluwer Academic Publishers, Dordrecht, Boston, London, 2001, pp. 193-211.

[28] J. Liouville. Extension au cas des trois dimensions de la question du tracé géographique, Note VI, pp. 609 - 617 in: G. Monge: Applications de l'analyse à la géométrie, Bachelier, Paris 1850.

[29] H. Malonek. Power series representation for monogenic functions in $\mathbb{R}^{m+1}$ based on a permutational product. Complex Variables Theory Appl., 15, 181-191 (1990).

[30] H. Malonek. Contributions to a geometric function theory in higher dimensions by Clifford analysis methods: Monogenic functions and M-conformal mappings, in: Clifford Analysis and its Applications ed. F. Brackx et al., Kluwer, NATO Sci. Ser. II, Math. Phys. Chem. 25, 213-222 (2001).

[31] H. Malonek and R. Almeida. A note on a generalized Joukowski transformation. Applied Mathematics Letters, 23, 1174-1178 (2010).

[32] H. Malonek, K. Gürlebeck, P. Cerejeiras, and U. Kähler. A quaternionic Beltrami type equation and the existence of local homeomorphic solutions. Applied Mathematics Letters, ZAA, 20, 17-34 (2001).

[33] J.B. Maricato, J.M. Machado and M.F. Borges. Quasiconformal transformations and hypercomplex functions, International Journal of Applied Math., 20 (2007), 691-702.

[34] I. Mitelman and M. Shapiro, Differentiation of the Martinelli-Bochner integrals and the notion of hyperderivability, Mathematische Nachrichten 172, no. 1, 1995, pp. 211-238.

[35] J. Morais. Approximation by homogeneous polynomial solutions of the Riesz system in $\mathbb{R}^{3}$, Ph.D. thesis, Bauhaus-Universität Weimar, 2009, 157 pages.

[36] J. Morais and K. Gürlebeck. Real-Part Estimates for Solutions of the Riesz System in $\mathbb{R}^{3}$, Complex Variables and Elliptic Equations, 18 pp, doi: 10.1080/17476933.2010.504838.

[37] S. Rickman. Quasiregular mappings, Ergebnisse der Mathematik und ihrer Grenzgebiete 26, SpringerVerlag, Berlin - Heidelberg - New York, 1993.

[38] M. Riesz. Sur les fonctions conjugués. Math. Z., 27, 218 âĂŞ244 (1927).

[39] G. Sansone. Orthogonal Functions. Pure and Applied Mathematics, Vol. IX. Interscience Publishers, New York, 1959.

[40] A. Sudbery. Quaternionic analysis. Math. Proc. Cambridge Phil. Soc. 85: 199-225 (1979).

[41] O. Teichmüller. Untersuchungen über konforme und quasikonforme Abbildung, Deutsche Math. 3 (1938), 621-678.

[42] J. Väisälä. On quasiconformal mappings of a ball, Ann. Acad. Sci. Fenn. Ser. A I Math. 304 (1961), 1-17.

[43] J. Väisälä. Lectures on n-dimensional quasiconformal mappings, Lecture Notes in Mathematics 229 (1971), Springer-Verlag. 
[44] M. Vuorinen. Conformal Geometry and Quasiregular Mappings, Lecture Notes in Mathematics 1319 (1988), Springer-Verlag.

[45] V. A. Zorich, The global homeomorphism theorem for space quasiconformal mappings, its development and related open problems, M. Vuorinen (ed.), Quasiconformal Space Mappings, Lecture Notes in Mathematics, 1508 (1992) pp. 132âĂŞ148. 